\title{
Ontogenia del esporangio y esporogénesis del licopodio Huperzia brevifolia (Lycopodiaceae) de las altas montañas de Colombia
}

\author{
Edgar Javier Rincón Barón ${ }^{1,2}$, Leidy Vivivana Gélvez Landazábal ${ }^{1}$, Helkin Giovany Forero \\ Ballesteros $^{1}$, Dagoberto Arrieta Prieto ${ }^{1} \&$ José Sergio Hleap $^{2}$ \\ 1. Laboratorio de Histotecnia, Escuela de Biología, Universidad Industrial de Santander, Bucaramanga, Colombia; vivi- \\ gela@gmail.com, helfos85@gmail.com,darrieta@uis.edu.co \\ 2. Departamento de Biología, Universidad del Valle, Valle del Cauca, Cali, Colombia; ejrbaron@gmail.com, sergiohle- \\ ap@yahoo.com
}

\author{
Recibido 10-II-2009. Corregido 06-V-2009. Aceptado 09-VI-2009.
}

\begin{abstract}
Sporangia ontogeny and sporogenesis of the lycopodium Huperzia brevifolia (Lycopodiaceae) from the high mountains of Colombia. Huperzia brevifolia is one of the dominant species of the genus Huperzia living in paramos and superparamos from the Colombian Andes. A detailed study of the sporangium's ontogeny and sporogenesis was carried out using specimens collected at $4200 \mathrm{~m}$ above sea level, in Parque Natural Nacional El Cocuy, Colombia. Small pieces of caulinar axis bearing sporangia were fixed, dehydrated, paraffin embedded, sectioned in a rotatory microtome, and stained using the common Safranin O-Fast Green technique; handmade cross sections were also made, stained with aqueous Toluidine Blue (TBO). The sporangia develops basipetally, a condition that allows observation of all the developmental stages taking place throughout the caulinar axis of adult plants. Each sporangium originates from a group of epidermal cells, axilar to the microphylls. These cells undergo active mitosis, and produce new external and internal cellular groups. The sporangium wall and the tapetum originate from the external group of cells, while the internal cellular group leads to the sporogenous tissue. Meiosis occur in the sporocytes and produce simultaneous types tetrads, each one giving rise four trilete spores, with foveolate ornamentation. During the sporangium ripening, the outermost layer of the wall develops anticlinally, and inner periclinal thickenings and the innermost one perform as a secretory tapetum, which persists until the spores are completely mature. All other cellular layers colapse. Rev. Biol. Trop. 57 (4): 1141-1152. Epub 2009 December 01.
\end{abstract}

Key words: Huperzia, Lycopodiaceae, Pteridophyta, sporangium ontogeny, sporogenesis, spores.

Las Lycopodiales son plantas pequeñas que tienen una distribución geográfica amplia y son cosmopolitas. Se conocen aproximadamente unas 500 especies agrupadas en diferentes géneros (Øllgaard 1992). Huperzia Bernh es el género mejor representado en el Neotrópico, con unas 300 especies (Øllgaard 1992), de las que unas 69 serían endémicas de los Andes de Colombia y Ecuador. Para Huperzia brevifolia (Grev. \& Hook.), Tryon \& Tryon (1982) proponen una distribución extensa, desde Alaska hasta la provincia de Tierra del Fuego, en Argentina; pero de trabajos más recientes, se concluye que estaría restringida a Costa Rica y los páramos de Colombia, Ecuador y Perú (Øllgaard 1987).

Los estudios en licopodiáceas son diversos, desde los de carácter florístico, sistemático o monográfico, como los de Santa (1989), Tardieu-Blot (1971), Toursarkissian (1971), Rolleri (1971, 1975, 1978, 1981, 1984, 1985 a-b, 1989), Rolleri \& Deferrari (1989), Øllgaard $(1987,1988,1992,1996)$, Tryon \& Stolze (1994), Murillo \& Harker (1990), Murillo \& Murillo (1999); morfológicos, como los de Rolleri (1972 a-b), Rolleri \& de la Sota (1972), 
Rolleri (1974, 1976, 1977 a-b, 1980), Øllgaard (1975), Rolleri \& Deferrari (1986), Santa (1986), hasta citológicos, como los de Rolleri (1982 a-b), entre otros.

La información sobre la biología reproductiva es escasa. Sikes (1908), dio a conocer las primeras descripciones sobre la estructura y organización de los esporangios en licopodiáceas; Wilce (1972) describió las esporas de Lycopodium L., Lycopodiella Holub y Huperzia, proponiendo grupos sobre la base de la ornamentación; Øllgarrd (1975) y Rolleri (1979) estudiaron la estructura y el modelo epidérmico de la pared del esporangio de especies incluidas hasta ese momento en Lycopodium, resaltando la importancia de estos caracteres en la sistemática. Uehara \& Kurita (1991), elaboraron el único estudio sobre la morfogénesis y ultraestructura de la pared de las esporas de licopodiáceas, tomando como modelo $L$. clavatum L. e incluyeron algunos aspectos relacionados con la formación de las esporas. Solé de Porta \& Murillo-Pulido (2005), describieron detalladamente las esporas de 24 especies de Huperzia, incluida H. brevifolia y, finalmente, Pita et al. (2006) describieron la morfología interna y externa de los microfilos y esporangios de 10 especies de Huperzia de Brasil, un trabajo amplio sobre aspectos anatómicos del género que, sin embargo, no hace referencia a los procesos de morfogénesis de los esporangios.

En el presente trabajo se presentan datos originales sobre el desarrollo del esporangio y la esporogénesis de $H$. brevifolia, con el fin de contribuir al conocimiento de la biología reproductiva de las licopodiáceas de Colombia y se comparan los resultados con Lycopodium, Lycopodiella y otras criptógamas, incluyendo briófitos y pteridófitos homosporados.

\section{MATERIALES Y MÉTODOS}

El material de $H$. brevifolia fue recolectado en localidades del Parque Nacional Natural El Cocuy (Boyacá, Colombia), a 4200m de altitud (6²2' 27.77' N, 72॰ 19' 01.92” W). El material de respaldo de este trabajo se encuentra depositado en el Herbario de la Escuela de Biología de la Universidad Industrial de Santander (UIS).

Se tomaron trozos de $5 \mathrm{~cm}$ de tallos portadores de esporangios de las porciones apicales de los ejes, se fijaron en FAA durante 24 horas y se deshidrataron en la serie clásica de alcoholes y se efectuaron dos cambios por xilol, aumentando los tiempos en cada etapa del procesamiento (Ruzin 1999). Luego de este tratamiento se tomaron fragmentos de $1 \mathrm{~cm}$ que fueron embebidos en Paraplast (Mc Cormick ${ }^{\circledR)}$ y se efectuaron cortes de $4-5 \mu \mathrm{m}$, con un micrótomo rotatorio 820 Spencer. Los cortes se colorearon con safranina-fastgreen y con la coloración cuádruple de Johansen (1940). También se efectuaron cortes a mano alzada y se colorearon con TBO 1\% acuoso. La observación se efectuó mediante un microscopio óptico Olympus CHS y los cortes se fotografiaron con una cámara digital Sony DSC-P71.

Todo el procesamiento y observación del material se llevó a cabo en el Laboratorio de Histotecnia de la Escuela de Biología de la Universidad Industrial de Santander.

\section{RESULTADOS}

Los ejemplares analizados de $H$. brevifolia tienen aproximadamente $25 \mathrm{~cm}$ de altura y las plantas presentan diferentes tonalidades de rojo (Fig. 1). Los esporangios se localizan en las axilas de los microfilos, en la zona de inserción con el eje caulinar y maduran de forma basípeta; los esporangios inmaduros están en el ápice de los tallos y los adultos en la base (Fig. 2).

El desarrollo de los esporangios comienza en un grupo de células epidérmicas, localizadas en la axila de los primordios apicales y de casi todos los microfilos adultos. Este grupo celular presenta divisiones periclinales y se establecen dos capas cuyas células tienen núcleos y nucléolos grandes, y citoplasma escaso (Fig. $3)$. En estas capas tiene lugar una activa división celular, que determina la formación de un agregado indiferenciado de células de aspecto juvenil, con núcleos grandes, varios nucléolos y citoplasma comparativamente escaso, con 


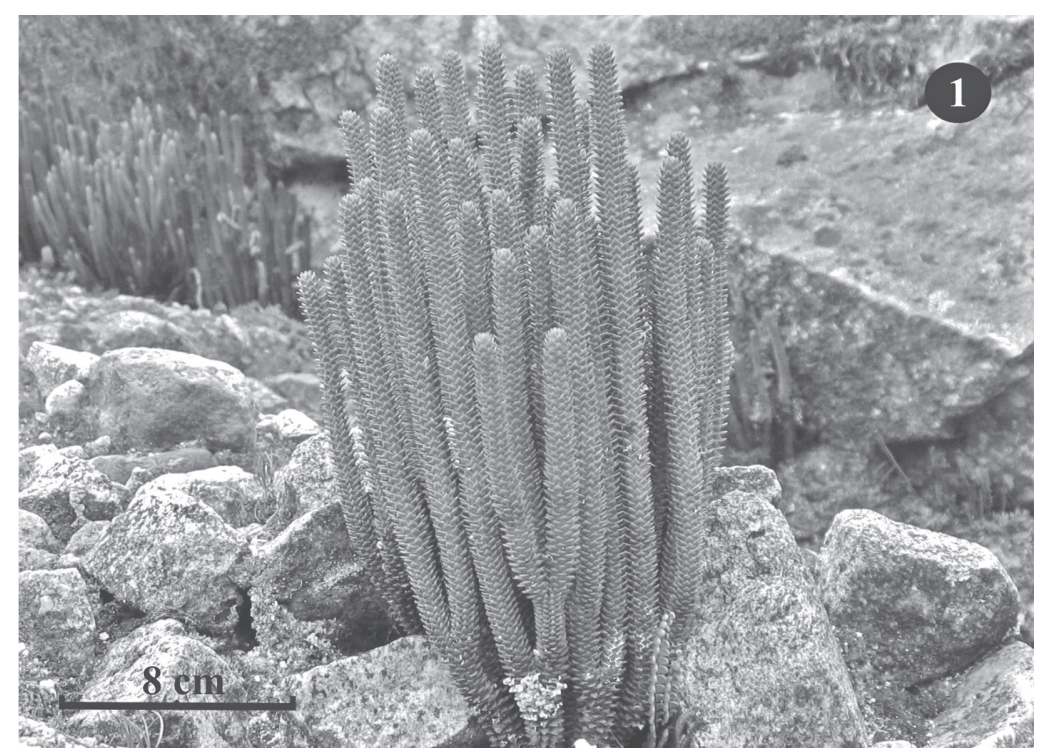

Fig. 1. Huperzia brevifolia, vista de la planta en roquedales del superpáramo.

Fig. 1. Huperzia brevifolia, view of a plant in rocky places of the superparamo.

granulación difusa (Fig. 4). En este agregado posteriormente se establecen dos grupos celulares bien definidos: uno interno, que forma el tejido esporógeno y uno externo (Fig. 5) que se diferenciará como pared del esporangio (Fig. 6). Finalmente, el esporangio queda constituido por la pared esporangial, el tapete que proviene del estrato interno de la pared del esporangio y las células madre o esporocitos, derivadas del tejido esporógeno, que se dividen activamente por mitosis (Fig. 7).

Los esporocitos se encuentran densamente agrupados en el esporangio (Fig. 7) y presentan una cubierta parcialmente asociada con la membrana celular (Fig. 8). Subsiguientemente, los esporocitos se hinchan, se separan (Fig. 9) y las cubiertas se degradan, liberándolos en la cavidad del esporangio (Fig. 10). En esta etapa tienen un núcleo grande, irregular, varios nucléolos y citoplasma vacuolado. Luego de perder la cubierta, los esporocitos experimentan la meiosis I, al final de la cual quedan en estado de díada, sin pared celular que separe los núcleos. En esta etapa es posible observar remanentes de las fibras del huso meiótico (Fig. 11).

Al iniciarse la meiosis II se restablece la cubierta de los esporocitos y finalmente se forma una tétrada joven con esporas en disposición tetraédrica (Fig. 12). En esta etapa se pueden observar también las fibras del huso y depósitos lipídicos. Las tétradas de $H$. brevifolia pasan por varias etapas de maduración, que se relacionan con el engrosamiento progresivo de las paredes de las esporas y con el surgimiento del patrón de ornamentación foveolada en la cara distal de las mismas (Figs. 13-14).

El tapete es uniestratificado y sus células tienen contorno rectangular (Fig. 15). Se diferencia durante la formación de los esporocitos, se mantiene íntegro durante las etapas de maduración de las tétradas y finalmente desaparece, luego del colapso de sus células. A medida que el esporangio madura, las capas celulares adyacentes al tapete degeneran progresivamente y se diferencian las células que constituyen el estrato celular externo, que adquieren engrosamientos en las paredes anticlinales y periclinales internas, estableciéndose 

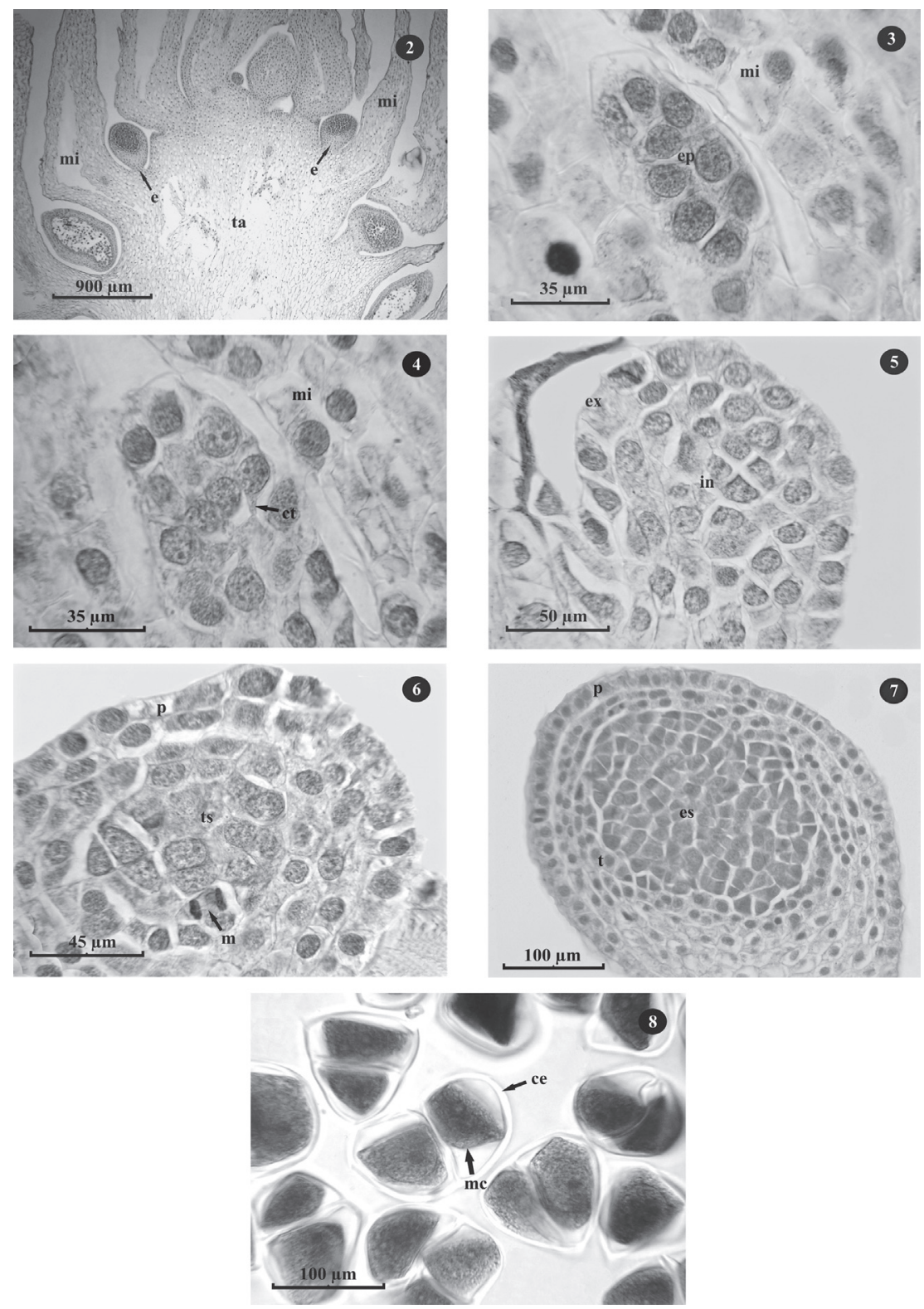

Fig. 2-8. Huperzia brevifolia, primeras etapas de la ontogenia del esporangio. 2. Corte longitudinal de la región apical del tallo. 3. Células epidérmicas iníciales del esporangio. 4-6. Desarrollo del esporangio, con aumento en el número de células por activas mitosis. 7. Esporangio diferenciado, con la pared, el tapete y los esporocitos. 8. Detalle de los esporocitos. ce: cubierta del esporocito, e: esporangios, ep: células epidérmicas, es: esporocitos, ex: zona externa, ct: citoplasma; in: zona interna, m: mitosis, me: membrana plasmática del esporocito, mi: microfilos, p: pared del esporangio, t: tapete, ta: tallo, ts: tejido esporógeno.

Fig. 2-8. Huperzia brevifolia, early ontogeny stages of the sporangium development. 2. Longitudinal sections of the apical part of the stem. 3.Initial epidermal cells of sporangium. 4-6. Sporangium development, with increasing number of cells due to high mitotic activity. 7. Adult sporangium, with tapetum and sporocytes. 8. Sporocyte details. ce: sporocyte coat, e: sporangium, ep: epidermal cells, es: sporocytes, ex: external zone, ct: cytoplasm, in: inner zone, m: mitosis, mi: microphyll, me: sporocyte's membrane, p: sporangium's wall, t: tapetum, ta: stem, ts: sporogenous tissue. 

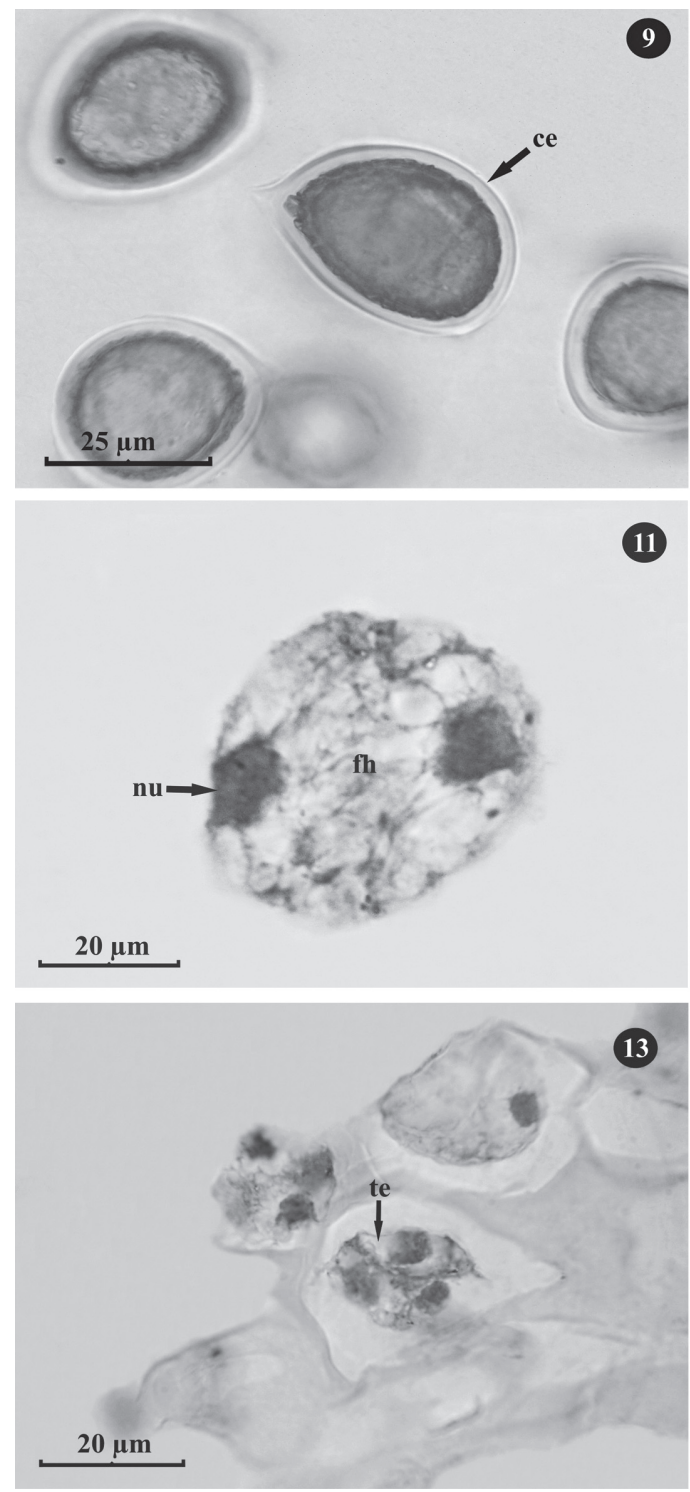
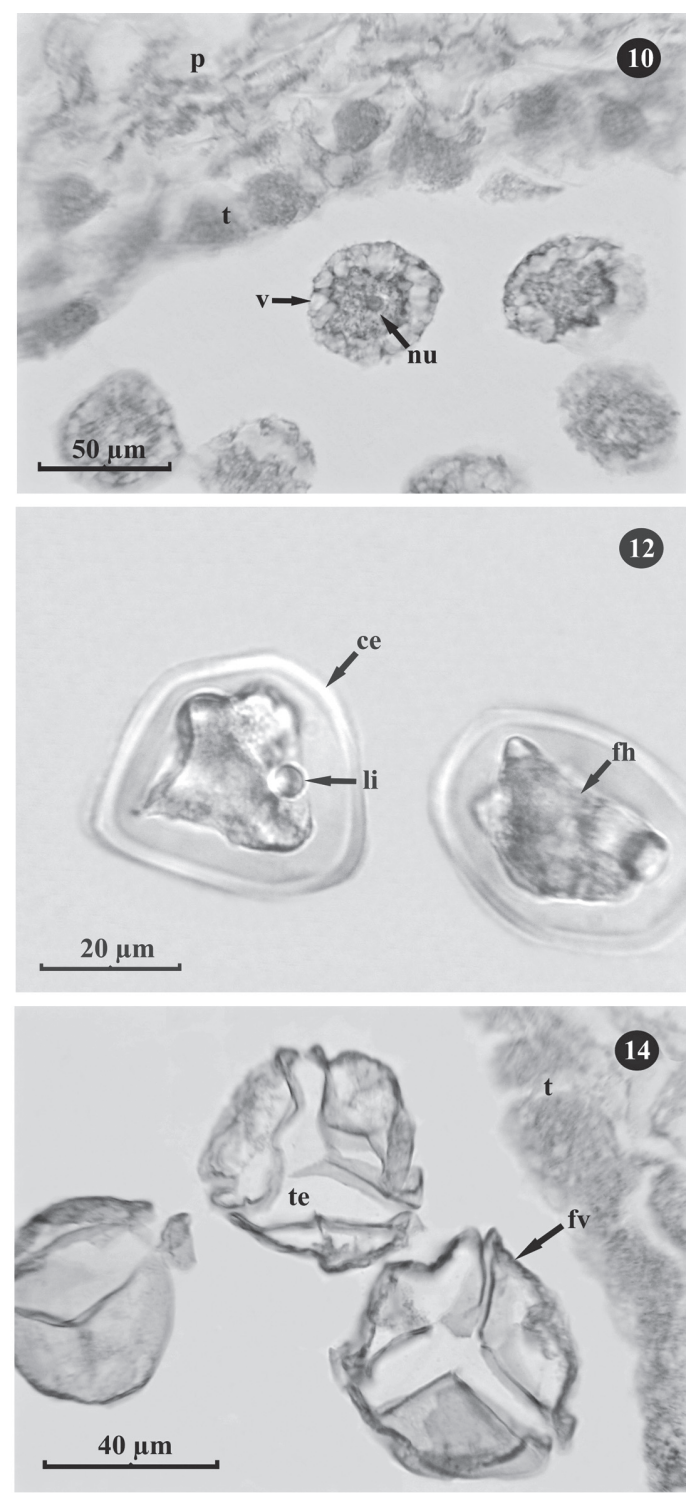

Fig. 9-14. Esporocitos maduros, estados meióticos y formación de tétradas de H. brevifolia. 9. Esporocitos hinchados envueltos por su cubierta y libres en la cavidad del esporangio. 10. Pérdida de la pared en esporocitos premeióticos y tapete. 11. Estado de díada al finalizar la meiosis I; se pueden observar las fibras del huso meiótico y la ausencia de pared entre los núcleos. 12. Tétradas en formación y restablecimiento de la cubierta del esporocito, durante la meiosis II. 13-14. Tétradas en diferentes etapas de maduración; se puede observar el engrosamiento progresivo de las paredes de las esporas y el inicio del patrón de ornamentación. ce: cubierta del esporocito, fh: fibras del huso, fv: foveola, li: depósitos lipídicos, nu: núcleo, p: pared del esporangio, t: tapete, te: tétrada, v: vacuolas.

Fig. 9-14. Huperzia brevifolia, mature sporocytes, meiotic stages, and formation of spore tetrad. 9. Swelled, loosed sporocytes within the sporangium cavity, covered by sporocyte coat. 10. View of the tapetum, and pre-meiotic sporocytes loosing the sporocyte coat. 11. End of the meiosis I, with a dyad stage, fibers of meiotic spindle and lacking of wall separating nuclei. 12. Developing tetrads and reestablishment of the sporocyte coat during meiosis II. 13-14. Tetrads in different developmental stages; view of the progressive thickening of the spore`s wall and the beginning of the ornamentation pattern. ce: sporocyte coat, fh: meiotic huse fibers, fv: foveole, li: lipidic deposits, nu: nucleus, p: sporangium's wall, t: tapetum, te: tetrad, v: vacuoles. 

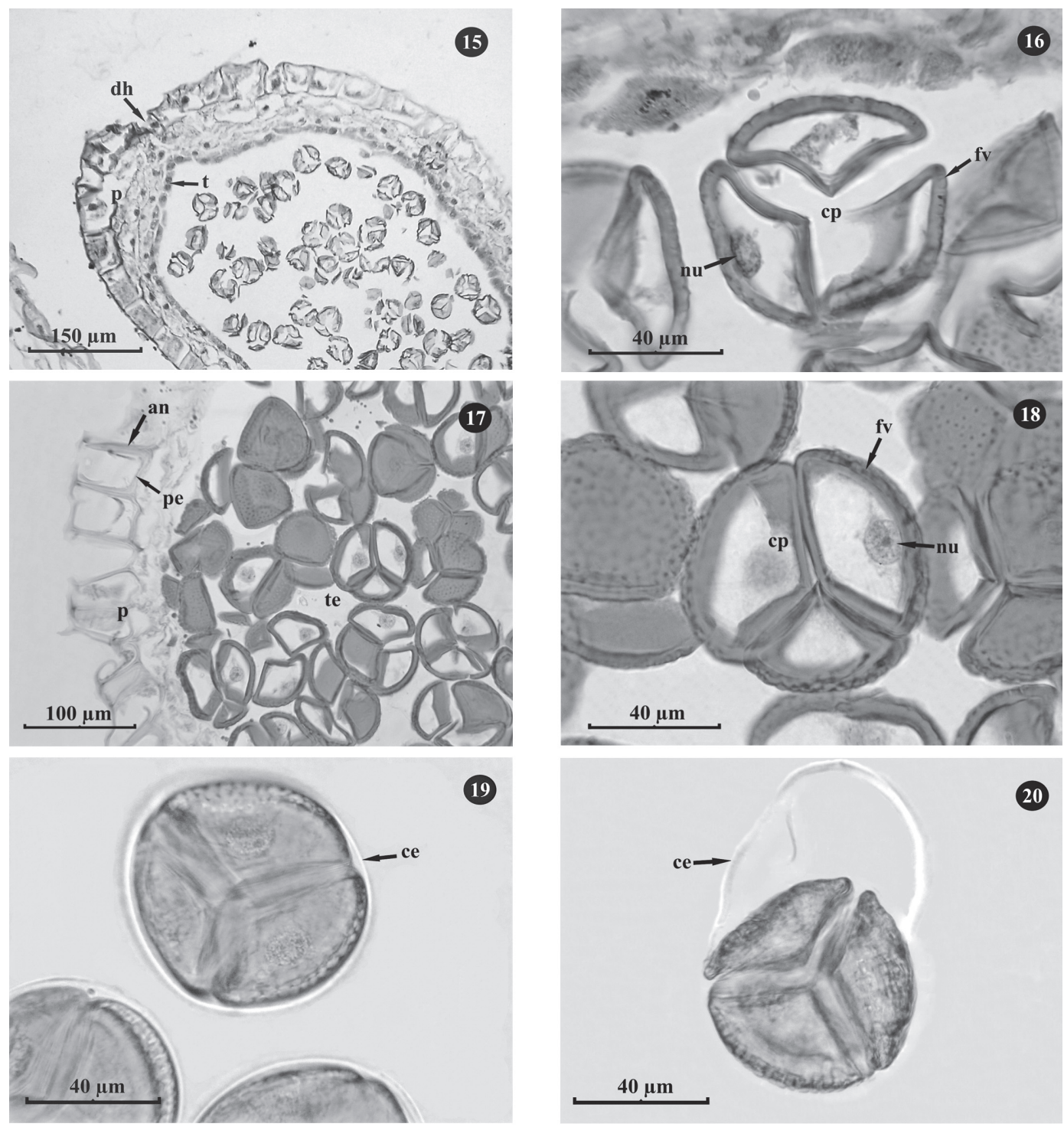

20
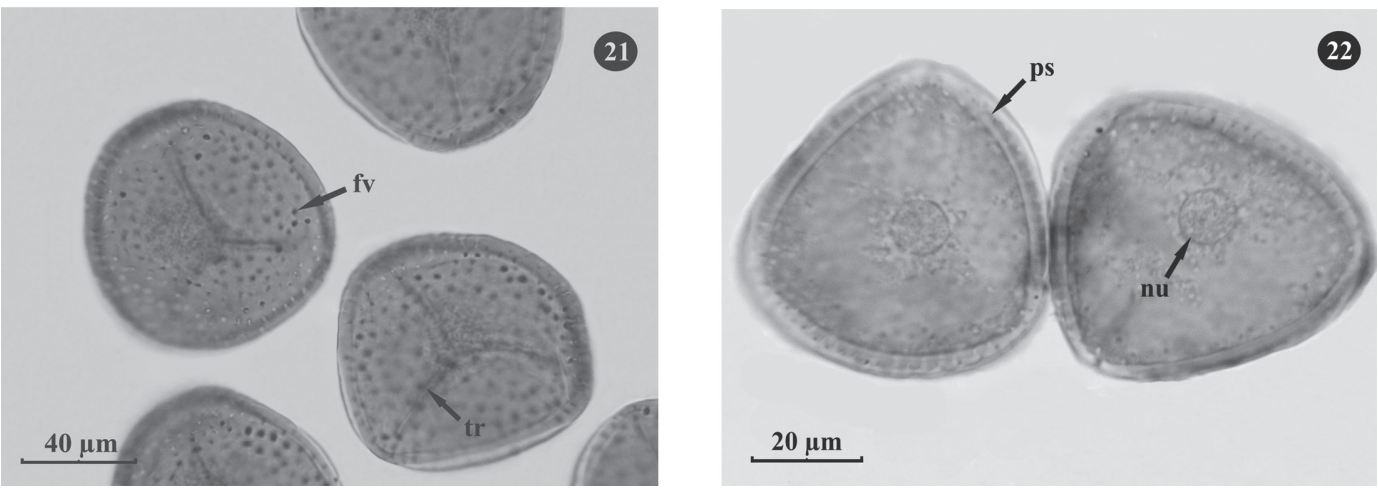
la pared definitiva del esporangio. Estas células varían en contorno, desde casi isodiamétricas en la proximidad de la zona de dehiscencia, hasta rectangulares, cerca al pie del esporangio (Figs. 16-17).

En tétradas maduras se pueden observar los núcleos de las esporas y los patrones de ornamentación de la pared esporal (Fig. 18). En preparaciones microscópicas permanentes no se aprecia la cubierta de los esporocitos, ya que ésta se pierde durante el procesamiento de las muestras, pero en preparados transitorios es posible apreciar que la cubierta del esporocito se conserva durante el proceso de maduración de las tétradas (Fig. 19), y sólo se rompe en el momento de la liberación de las esporas (Fig. 20).

Finalmente, las esporas triletes son liberadas de la tétrada en la cavidad del esporangio. Presentan una cara distal foveolada (Fig. 21) y una proximal lisa (Fig. 22), en la que se localiza la lesura con tres brazos. Los esporangios maduros presentan dos valvas iguales, tienen un diámetro promedio de 1.5-2.5 $( \pm 0.70) \mathrm{mm}$ y un pie corto. Las esporas se liberan por la abertura que se forma entre las dos valvas del esporangio, en la zona formada por células isodiamétricas más pequeñas que las adyacentes y con paredes marcadamente engrosadas. En ninguna etapa del desarrollo se observó tejido vascular, ni células de transfusión asociadas a los esporangios.

\section{DISCUSIÓN}

El desarrollo de los esporangios de $H$. brevifolia es basípeta, con los esporangios maduros localizados en la porción inferior del eje caulinar y los inmaduros en el ápice. Todas las etapas de desarrollo se pueden encontrar sobre un mismo eje caulinar adulto. Esta condición también fue señalada por Rolleri (1972 a-b) y Uehara \& Kurita (1991) para Lycopodium clavatum y por (Øllgard 1987) para otras licopodiáceas como Phylloglosum Kunze y Lycopodiella, algo que sugiere cierta uniformidad en los géneros en cuanto a la ontogenia esporangial.

Los esporangios de las licopodiáceas se originan sobre la cara adaxial de los microfilos o de los esporofilos jóvenes, cerca de la inserción de éstos en el eje caulinar. En H. brevifolia se da la primera condición debido a la ausencia de estróbilos definidos. En Lycopodium, durante las etapas iniciales del desarrollo, los esporangios también se localizan en la inserción de los microfilos o de los esporofilos; mientras que en Lycopodiella (Rincón Barón et al., en prep.), los esporangios maduros se localizan en la región media adaxial de los esporofilos.

Fig. 15-22. Tétradas en diferentes etapas de maduración, tapete, dehiscencia y esporas de H. brevifolia. 15. Esporangio maduro; se observa el tapete (estrato interno), las células de la pared y la dehiscencia. 16-18. Tétradas en maduración; se observa el engrosamiento progresivo de las paredes de las esporas con el patrón de ornamentación más notable, el núcleo de las esporas desplazado hacia la cara distal y evidentes engrosamientos de las paredes anticlinales y periclinal interna de las células de la pared. 19-20. Tétradas observadas en preparaciones elaboradas a mano alzada, en las que se observa la cubierta del esporocito y su ruptura para liberar las esporas. 21. Esporas en vista distal, ornamentación foveolada y lesura en la cara proximal. 22. Esporas en vista proximal con la superficie lisa. an: anticlinal, cd: cara distal, ce: cubierta del esporocito, cp: cara proximal, dh: dehiscencia, fv: foveola, nu: núcleo, p: pared del esporangio, pe: periclinal, ps: pared de la espora, t: tapete, te: tétradas, tr: trilete.

Fig. 15-22. Huperzia brevifolia: view of spore tetrads in different developmental stages, with tapetum, dehiscence process in progress, and spores. 15. A mature sporangium, beginning its dehiscence, with their layers of wall and tapetum. 16-18. Development of spores: tetrad and spores with wall thickening and clear ornamentation can be seen; the nucleus of spores mostly located towards the distal face, anticlinal and inner periclinal wall thickenings becoming more evident. 19. Tetrad with sporocyte coat. 20. Rupture of sporocyte coat, and liberated spores 21. Distal and proximal views of spores with foveolate ornamentation, and lesurae. 22. Proximal view of two spores, with smooth wall surface.

an: anticlinal, cd: distal face, ce: sporocyte coat, cp: proximal face, di: dehiscence, fv: foveole, nu: nucleus, p: sporangium's wall, pe: periclinal, ps: spore's wall, t: tapetum te: tetrads, tr: trilete. 
Rolleri (1972 a), describió la morfología comparada de algunas especies de Lycopodium, incluyendo las etapas de morfogénesis de los esporangios y observó que en el inicio de éstas, las células epidérmicas que los forman se dividen periclinalmente para producir dos capas de células: una basal que origina el pedicelo y otra superficial que origina la pared del esporangio $\mathrm{y}$ el tejido esporógeno. Luego las divisiones periclinales y anticlinales en estas capas determinan el aumento del número de células que formarán el esporangio maduro, una condición que es similar a la observada en $H$. brevifolia.

Durante la ontogenia del esporangio de $H$. brevifolia, se observó que los esporocitos se forman a partir de la diferenciación del tejido esporógeno y sin la presencia de una cubierta del esporocito ("sporocite coat"). Rolleri (1972 a) describió un patrón similar para las especies de Lycopodium del noreste de Argentina, aunque esta autora no utilizó el término "cubierta del esporocito", que fue propuesto por Pettit \& Jermy (1974), para designar la cubierta superficial que se forma sobre las células madre de los pteridófitos en general. Uehara \& Kurita (1991), señalan que en Lycopodium los esporocitos se encuentran densamente empaquetados en el tejido esporógeno de la cavidad esporangial y se comunican entre sí por medio de la cubierta mencionada; a medida que maduran, la cubierta se disuelve permitiendo la liberación de los esporocitos premeióticos en el fluido de la cavidad esporangial. Esta condición también se observó en $H$. brevifolia y ha sido registrada para algunos briófitos (Mueller 1974). En Lycopodiella, en cambio, la cubierta original de los esporocitos persiste durante todo el proceso de esporogénesis, lo que podría sugerir que no es tan importante para el transporte de materiales precursores que formarán la pared de las esporas, y que existirían otros mecanismos de transporte entre el tapete y aquellas (Rincón Barón et al., en prep.). Mueller (1974), observó que la degradación de la cubierta de los esporocitos en Fissidens limbatus Sullivant los prepara para la meiosis posterior y facilita el proceso de transporte entre el tapete y las esporas. Según Uehara \& Kurita (1991) esto se cumple para L. clavatum. En H. brevifolia se podría presentar un fenómeno similar. Los esporocitos premeióticos de $H$. brevifolia son morfológicamente diferentes de los juveniles o de los que están en etapas tempranas del desarrollo, debido a la ausencia de la cubierta, la presencia de un núcleo irregular con varios nucléolos y citoplasma con abundantes depósitos lipídicos. Estos caracteres ultrastructurales fueron descritos por Uehara \& Kurita (1991) para $L$. clavatum y por Lugardon (1990) y Uehara \& Kurita (1989), para otros pteridófitos, e indican la gran actividad metabólica de estas células en relación con el almacenamiento de sustancias de reserva y la producción de precursores que intervendrán en la formación del endosporio.

En los esporocitos de $H$. brevifolia se presenta meiosis simultánea y no se desarrolla una pared que separe los núcleos de la díada, una vez terminada la meiosis I. Furness et al. (2002), hacen referencia a la presencia de este tipo de comportamiento meiótico en briófitos y pteridófitos, relacionándola con los grupos que producen esporas triletes, en contraste con los grupos de pteridófitos que producen esporas monoletes y que, generalmente, presentan división sucesiva. Estos autores señalan también que la división simultánea, comúnmente hallada en briófitos y pteridófitos, se puede considerar un carácter ancestral y que la división sucesiva ha surgido de manera independiente a lo largo del proceso evolutivo en ambos grupos.

Rolleri (1972a) observó, en diferentes especies de Lycopodium, que la pared del esporangio está formada inicialmente por seis a siete capas celulares con citoplasma granular y grandes núcleos. En el curso del desarrollo, el número de capas parietales se reduce a cuatro. En $H$. brevifolia se encontraron cuatro a cinco capas: la más externa forma la pared definitiva del esporangio y la más interna, el tapete. Las demás capas intermedias degeneran a medida que se desarrolla el esporangio, una condición similar a la descrita por Uehara \& Kurita (1991) para L. clavatum.

El estrato celular externo de la pared del esporangio de $H$. brevifiolia presenta engrosamientos lignificados en forma de U, 
característica descrita por Øllgaard (1975) para las licopodiáceas en general. Estos engrosamientos se desarrollan a medida que el esporangio madura y le confieren resistencia suficiente para contener el número creciente de esporas que sólo se liberarán en la madurez. Asimismo, es posible que faciliten su liberación, actuando como un efecto de resorte en la dispersión de las esporas a cierta distancia de la planta original. Sin embargo, poco se sabe de estos mecanismos, excepto los descritos para helechos leptosporangiados (Slosson 1906, Bierhorst 1971, Moran 2004) y no existen indicios de que ocurran en licopodiáceas.

El tapete es una estructura omnipresente en las plantas. Se relaciona con los procesos de esporogénesis en briófitos y pteridófitos, así como con la microsporogénesis de gimnospermas y angiospermas; contribuye con los procesos de dispersión (Pacini \& Franchi 1993), con la generación de sustancias de reserva y con la formación de precursores biosintéticos del esporodermo y de la pared de los granos de polen (Lugardon 1990, Polowick \& Sawhney 1993, Parkinson \& Pacini 1995, Pacini 1997, Furness \& Rudall 2001, Furness 2008). Los dos tipos, el secretor (celular) y el plasmodial se encuentran ampliamente distribuidos en las plantas terrestres (Parkinson \& Pacini 1995, Furness 2008). El tapete de H. brevifolia es de tipo secretor, se origina a partir de la capa celular interna de la pared del esporangio y se mantiene hasta que finalmente degenera cuando las esporas alcanzan la madurez. Uehara \& Kurita (1991), establecieron que la función del tapete en $L$. clavatum es nutricia en relación con las esporas en desarrollo y contribuye a la formación del esporodermo. Dada la uniformidad ontogenética antes mencionada, se puede asumir que el tapete de $H$. brevifolia cumple funciones similares.

Los esporangios de $H$. brevifolia presentan dos valvas, como en la mayoría de las licopodiáceas y la liberación de las esporas tiene lugar por la separación de las valvas a través de un área específica como sugirió Pita et al. (2006), para las especies de Huperzia de Brasil. No presentan vascularización, como observaron
Pita et al. (2006), quienes reportaron la presencia de células parenquimáticas de paredes más delgadas, comparadas con las otras células de la corteza del tallo. Gensel (1992), sugirió que en licófitos existen células lignificadas en el pedicelo, que hacen contacto con el tejido vascular del esporofilo y que funcionarían como un tejido de transfusión, pero en el presente estudio no se observaron.

Las esporas son foveoladas, dato que ya fue citado por Wilce (1972) y Solé de Porta \& Murillo-Pulido (2005).

\section{AGRADECIMIENTOS}

A Cristina Rolleri (Laboratorio de Estudios de Anatomía Vegetal Evolutiva y Sistemática -LEAVES-, Facultad de Ciencias Naturales y Museo de La Plata, Argentina), por la lectura crítica y valiosas sugerencias al documento final. A Jefferson Prado (Instituto de Botânica, Divisão de Fitotaxomonia, São Paulo, Brasil), por facilitarnos parte de la bibliografía. A Heiber Cárdenas, Jaime Cantera (Universidad del Valle, Departamento de Biología, Cali, Colombia), Blanca Pérez García (Departamento de Biología, Botánica Estructural y Sistemática Vegetal, Universidad Autónoma Metropolitana Iztapalapa, México), a Luz Amparo Triana (Universidad Nacional de Colombia) y a Beatriz Helena Guerra (UIS), por sus comentarios y oportunas sugerencias. A la Vicerrectoría Académica y de Investigación y Extensión de la Universidad Industrial de Santander, por el apoyo brindado. A Fabio Muñoz y al Sistema de Parques Nacionales Naturales (SPNN), por facilitarnos el acceso a la zona y permitir las recolecciones.

\section{RESUMEN}

Se describe la ontogenia y la esporogénesis en $H$. brevifolia, en material recolectado en el Parque Nacional Natural El Cocuy (Boyacá-Colombia) a 4200m de altitud. Los esporangios se desarrollan de forma basípeta sobre el eje caulinar: los iniciales y juveniles se localizan en el ápice y los adultos a maduros, en la base. El desarrollo se inicia a partir de un grupo de células epidérmicas localizadas en las axilas que forman los microfilos con el eje caulinar. Estas 
células se dividen activamente por mitosis formando una masa celular externa y otra interna. La primera da origen a la pared del esporangio, de varios estratos celulares; de éstos, el estrato externo desarrolla engrosamientos en las paredes anticlinales y en la periclinal interna. El estrato celular interno se diferencia para formar el tapete secretor. Los demás estratos celulares de la pared se degradan durante la maduración del esporangio. La masa celular interna da origen al tejido esporógeno que forma los esporocitos, que experimentan la meiosis I hasta la etapa de díada. La meiosis II concluye con la formación de tétradas, constituidas por esporas en disposición tetraédrica. Las esporas son foveoladas con abertura trilete y son liberadas del esporangio a través de la dehiscencia.

Palabras Clave: Huperzia, Lycopodiaceae, Pteridophyta, ontogenia del esporangio, esporogénesis, esporas.

\section{REFERENCIAS}

Bierhorst, D. 1971. Morphology of vascular plants. The Macmillan Company. Nueva York, EEUU.

Furness, C. A. \& P.J Rudall. 2001. The tapetum in basal angiosperms: early diversity. Int. J. Plant Sci. 162: 375-392.

Furness, C.A., P.J. Rudall \& B.F. Sampson. 2002. Evolution of microsporogenesis in angiosperms. Int. J. Plant Sci. 163: 235-260.

Furness, C. A. 2008. A review of the distribution of plasmodial and invasive tapeta in eudicots. Int. J. Plant Sci. 169: 207-223.

Gensel, P.G. 1992. Phylogenetic relantionships of the zosterophylls and lycopsids: evidence from morphology, paleoecology, and cladistic methods of inference. Ann. Mo. Bot. Gard. 79: 450-473.

Johansen, D. A. 1940. Plant microtechnique. McGrawHill. Nueva York, EEUU.

Lugardon, B. 1990. Pteridophyte sporogenesis: a survey of spore wall ontogeny and fine structure in a polyphyletic plant group. p. 95-120. In S. Blackmore \& R.B. Knox (eds). Microspores: Evolution and ontogeny. Academic Press, Londres, Inglaterra.

Moran, R. C. 2004. A natural history of ferns. Timber Press, Portland, Oregon, EEUU.

Mueller, D. M. 1974. Spore wall formation and chloroplast development during sporogenesis in the moss Fissidens limbatus. Am. J. Bot. 61: 525-534.

Murillo, M. T. \& M. Harker. 1990. Helechos y plantas afines de Colombia. Acad. Colomb. Cienc. Exac. Fis.
Nat. Colección Jorge Álvarez Lleras. 12. Bogotá, Colombia.

Murillo, M.T. \& J. Murillo. 1999. Pteridófitos de Colombia I. Composición y distribución de las lycopodiaceae. Rev. Acad. Col. Ciencias. XXIII: 19-38.

Øllgaard, B. 1975. Studies in Lycopodiaceae, I. Observations on the structure of the sporangium wall. Amer. Fern J. 65: 19-27.

Øllgaard, B. 1987. A revised classification of the Lycopodiaceae. Lat. Opera Bot. 92: 153-178.

Øllgaard, B. 1988. Lycopodiaceae. In G. Harling \& L. Andersson (eds). Flora of Ecuador. No. 33 Arlöv Berlings.Quito, Ecuador.

Øllgaard, B. 1992. Neotropical Lycopodiaceae-an overview. Ann. Mo. Bot. Gard. 79: 687-717.

Øllgaard, B. 1996. Neotropical Huperzia (Lycopodiaceae)distribution of species richness, p. 93-100. In J.M. Camus, M. Gibby \& R.J. Johns (eds). Pteridology in Perspective. Royal Bot. Gardens, Kew, Inglaterra.

Pacini, E. \& G. G. Franchi. 1993. Role of the tapetum in pollen and spore dispersal. Plant Syst. Evol. 7: 1-11.

Pacini, E. 1997. Tapetum character states: analytical keys form tapetum types and activities. Can. J. Bot.75: 1448-1459.

Parkinson, B.M \& E. Pacini. 1995. A comparison of tapetal structure and function in pteridophytes and angiosperms. Plant Syst. Evol.198: 55-88.

Pettitt, J. M \& A.C Jeremy. 1974. The surface coats on spores. Biol. J. Linn. Soc. Lond. 6: 245-257.

Pita, P., N. De Menezes \& J. Prado. 2006. Morfología externa e interna das folhas vegetativas, esporofilos e esporângios de espécies de Huperzia Bernh. (Lycopodiaceae-Pteridophyta) do Brasil. Rev. Bras. Bot. 29: 115-131.

Polowick, P.L. \& V.K. Sawhney. 1993. Differentiation of the tapetum during microsporogenesis in tomato (Lycopersicon esculentum Mill.) with special reference to the tapetal cell wall. Ann. Bot. 72: 595-905.

Rolleri, C.H. 1971. Dos nuevas especies de Lycopodium (Lycopodiaceae-Pteridophyta) para el noroeste argentino. Darwiniana 16: 133 - 139.

Rolleri, C. 1972a. Morfología comparada de las especies de "Lycopodium" (Pteridophyta-Lycopodiaceae) para el noroeste argentino. Rev. Mus. La Plata. 12: 223-317. 
Rolleri, C.H. 1972b. Sobre la presencia y naturaleza de la endodermis en especies de Lycopodium L. s. str. Bol. Soc. Argent. Bot. 14: 365-369.

Rolleri, C.H. 1974. Morfología de Lycopodium fuegianum Roivanen, Lycopodiaceae- Pteridophyta. Bol. Soc. Argent. Bot. 15: 365- 383 .

Rolleri, C.H. 1975. A new species of Lycopodium L. from the Peruvian Andes. Amer. Fern J. 65: 3-6.

Rolleri, C.H. 1976. Acerca de los conductos glandulares en hojas de Lycopodium alopecuroides LLycopodiaceae-Pteridophyta. Bol. Soc. Argent. Bot. 16: 397-405.

Rolleri, C.H. 1977a. Correlation of morphology and geographical distribution in Lycopodium saururus Lam. American Fern Journal 67: 109-120.

Rolleri, C.H. 1977b. Estudios morfológicos y sistemáticos en la sección Crassistachys Herter del género Lycopodium L.:L. crassum Humb.\& Bonpld. ex Willd. y L. saururus Lam. Rev. Mus. La Plata, Obra del Centenario III (Botánica): 97-110.

Rolleri, C.H. 1978. Dos nuevas especies de Lycopodium L. para los Andes tropicales. Hickenia 1: 142-148.

Rolleri, C.H. 1979. Análisis morfológico y revisión sistemática de las especies andino- patagónicas del género Lycopodium L., Lycopodiaceae-Pteridophyta. Physis (Buenos Aires), C, 38, 95: 39-62.

Rolleri, C.H. 1980. Estudios morfológicos y sistemáticos en la sección Crassistachys Herter del género Lycopodium L. L. brevifolium Hook. \& Grev. y L. rufescens Hook. Bol. Soc. Argent. Bot. 19: 243-254.

Rolleri, C.H. 1981. Sinopsis de las especies de Lycopodium L. (LycopodiaceaePteridophyta) de la sección Crassistachys Herter. Rev. Mus. La Plata N. S. Bot. 12: $61-114$

Rolleri, C.H. 1982a. El número cromosómico Lycopodium saururus Lam. (Lycopodiaceae-Pteridophyta) y sus implicancias sistemáticas. Rev. Mus. La Plata N. S. Bot. 13: 115-118.

Rolleri, C.H. 1982b.Elnúmero cromosómico de Lycopodium clavatum L. subsp. clavatum (LycopodiaceaePteridophyta) y sus implicancias sistemáticas. Rev. Mus. La Plata N. S. Bot.13: 119-122.

Rolleri, C.H. 1984. Notas nomenclaturales y taxonómicas en la sección Crassistachys Herter del género Lycopodium L.: L. cruentum Spring. Rev. Mus.La Plata N. S. Bot. 14: 45-63.

Rolleri, C.H. 1985a. Notas nomenclaturales y taxonómicas en la sección Crassistachys Herter del género
Lycopodium L., II. Rev. Mus.La Plata N. S. Bot. 14: 1-9.

Rolleri, C.H. 1985b. Estudios morfológicos y sistemáticos en la sección Crassistachys Herter del género Lycopodium L. Rev. Mus. La Plata N. S. Bot. 13: 189-196.

Rolleri, C.H. 1989. Huperzia recurvifolia Rolleri (Huperziaceae-Roth., Pteridophyta), una nueva especie de Brasil. Nat. Mus. La Plata N. S. Bot. 21: 209-213.

Rolleri, C.H. \& A.M. Deferrari. 1986. Modelos epidérmicos y otros caracteres foliares en la sistemática y ecología de Lycopodium L., sección Crassistachys Herter. Rev. Mus. La Plata N. S. Bot. 14: 65-87.

Rolleri, C.H. \& E.R. De La Sota. 1972. Sobre la presencia de Lycopodium magellanicum (Pal. Beauv.) Sw. en el noroeste de Argentina. Bol. Soc. Argent. Bot. 14:198-202.

Rolleri, C.H. \& A.M. Deferrari. 1989. Nota sobre la transferencia de especies del género Lycopodium L. (Lycopodiaceae-Mirbel) al género Huperzia Bernh. (Huperziaceae Roth.). Rev. Mus. La Plata N. S. Bot. 2: $151-157$.

Ruzin, S. E. 1999. Plant microtechnique and microscopy. Oxford University, NuevaYork, EEUU.

Santa, J. 1986. Anatomía del tallo y suscripción genérica en Licopodiaceae. Actual. Biol. 15: 102-116.

Santa, J. 1989. Estudio sobre la distribución de algunas licopodíneas de Colombia. Actual. Biol. 18: 95-103.

Slosson, M. 1906. How ferns grow. Henry Holt \& Co, Nueva York, EEUU.

Solé de Porta, N. \& M.T. Murillo-Pulido. 2005. Estudio palino-botánico de algunas especies de Pteridophyta de Colombia. Rev. Acad. Colomb. Cienc. 29: 183-218.

Sykes, M.G. 1908. Notes on the morphology of the sporangium-bearing organs of the lycopodiaceae. New Phytol. 7: 41-60.

Tardieu-Blot, M.-L., 1971. Lycopodiacées (Lycopodiaceae), 13 p. 8-9. In Keraudren-Aymonin, M. Flore de Madagascar et des Comores, familles 13 et 13 bis. Museum National d'Histoire Naturelle. Paris, Francia.

Toursarkissian, M. 1971. Las lycopodiaceae del noroeste de Argentina-estudio sistemático y anatómico. Ann. Acad. Bras. Ciencias. 43: 191-207.

Tryon, R. \& A. Tryon. 1982. Ferns and allied plants with special reference to tropical America. Springer, Nueva York, EEUU. 
Tryon, R.M. \& Stolze, R.G. 1994. Pteridophyta of Peru. Part VI. 22. Marsileaceae-21. Isoetaceae. Fieldiana Botany N. S. 34: 1-190.

Uehara, K \& S. Kurita. 1989. An ultrastructural study of spore wall morphogenesis in Equisetum arvense. Am. J. Bot. 76: 939-951.
Uehara, K \& S. Kurita.1991. Ultrastructural study on spore wall morphogenesis in Lycopodium clavatum (Lycopodiaceae). Am. J. Bot. 78: 24-36.

Wilce, J. H. 1972. Lycopod spores, I. General spore patterns and the generic segregates of Lycopodium. American Fern Journal 62: 365-79. 